\title{
From SARS-CoV-2 infection to COVID-19 disease: a proposed mechanism for viral spread to the lower airway based on in silico estimation of virion flow rates
}

\author{
Saikat Basu ${ }^{1,2, *}$ and Arijit Chakravarty ${ }^{3, *}$ \\ ${ }^{1}$ Department of Mechanical Engineering, South Dakota State University, Brookings, SD, United States \\ ${ }^{2}$ Department of Otolaryngology / Head and Neck Surgery, School of Medicine - University of North Carolina, \\ Chapel Hill, NC, United States \\ ${ }^{3}$ Fractal Therapeutics, Cambridge, MA, United States \\ *Email: Saikat.Basu@sdstate.edu; arijit@fractaltx.com
}

\section{ABSTRACT}

While the nasopharynx in the upper respiratory airway is the dominant initial infection site for SARS-CoV2, the physiologic mechanism that launches the infection in the lower airway is still not well-understood. Based on the rapidity with which SARS-CoV-2 infection progresses to the lungs, it has been conjectured that the nasopharynx acts as the seeding zone for subsequent contamination of the lower airway via aspiration of virus-laden boluses of nasopharyngeal fluids. In this study, we examine the plausibility of this proposed mechanism. To this end, we have developed computational fluid mechanics models of the inhalation process in two medical imaging based airway reconstructions and have quantified the nasopharyngeal liquid volume ingested into the lower airspace during each aspiration. The numerical predictions are validated by comparing the number of projected aspirations (approximately 2 - 4) during an eight-hour sleep cycle with prior observational findings of 3 aspirations in human subjects. Extending the numerical trends on aspiration volume to earlier records on aspiration frequency for the entire day indicates a total aspirated nasopharyngeal liquid volume of $0.3-0.76 \mathrm{ml}$ per day. We then used sputum assessment data from hospitalized COVID-19 patients to estimate the number of virions that are transmitted daily to the lungs via nasopharyngeal liquid boluses. For mean sputum viral load, our modeling projects that the number of virions penetrating to the lower airway per day will range over $2.1 \times 10^{6}-5.3 \times 10^{6}$; for peak viral load, the corresponding number of penetrating virions hovers between $7.1 \times 10^{8}-17.9 \times 10^{8}$. These findings fill in a key piece of the mechanistic puzzle of the progression from SARS-CoV-2 infection of the nasopharynx to the development of COVID-19 disease within a patient, and point to dysphagia as a potential underlying risk factor for COVID-19. The findings also have significant practical implications in the design of COVID-19 prophylactics and therapeutics that aim to constrain the pathogenic progress of the disease within the limits of the upper airway.

Keywords: SARS-CoV-2 infection, COVID-19, Nasopharyngeal Transmission, Lower Airway Infection, Computational Fluid Dynamics, Dysphagia

\section{Introduction}

Severe acute respiratory syndrome coronavirus 2 (SARS-CoV-2), the causative agent for coronavirus disease 2019 (COVID-19), has been linked ${ }^{1,2}$ to a remarkable pattern of relatively high infectivity in ciliated epithelial cells along the nasal passage lining in the upper airway, moderate infectivity in cells lining the throat and bronchia, and relatively low infectivity in lung cells. Such viral tropism is governed by the abundance of angiotensin-converting enzyme 2 (ACE2), a single-pass type I membrane protein that is exploited by the viral spike protein binding as a gateway for cellular entry. ACE2 is abundant on ciliated epithelial cells, but is highly expressed in only a smaller subset of the 
alveolar cells in the lower airway. The findings are nonetheless for in vitro samples; virus-laden droplets deposited along the anterior nasal airway might not be so effective at launching an infection despite the presence of ciliated cells, as the thicker mucus layer there provides some level of protection against virus invasion and infection ${ }^{3}$. Hence, the nasopharynx, which is the region in the upper airway posterior to the septum comprising the superior portion of the pharynx, has been postulated to be the main initial infection site for SARS-CoV-2. Efficacy of nasopharyngeal swabs over oropharyngeal swabs for accurate detection ${ }^{4}$ of COVID-positive cases supports this hypothesis. Based on the brisk pace at which lower airway infections ensue following the emergence of initial symptoms ${ }^{1}$, it may be conjectured that the nasopharynx (marked in Fig. 1, see Panels (a)-(f)) acts as the seeding zone for subsequent spread of the disease to the lungs via lower airway aspiration of virus-laden boluses of nasopharyngeal fluids.

While this conjectured mechanism is superficially plausible, a key unanswered question is whether the rate of virion flow from the initial site of infection to the lungs would be sufficiently high to account for a second infection site. In this report, we have combined earlier data on aspiration trends ${ }^{5,6}$ with virological assessments of sputum in COVID-positive patients ${ }^{7}$ and computational findings on the flow physics variables in anatomically realistic airway models $^{8}$ - to quantify bolus-borne virion transmission rates from the nasopharynx into the lower airway.

\section{Materials and Methods}

\subsection{Frequency and quantification of pharyngeal aspiration}

Aspiration of upper airway secretions acts as a major carrier of pathogens to the lower airway, and the phenomenon, fortunately enough given the current pandemic, has been studied in great detail over the last few decades. As reported in the late-1990s $\mathrm{s}^{5}$, aspirated pharyngeal liquid volume during sleep ranges from $0.011 \mathrm{ml}$ to $0.129 \mathrm{ml}$, measured through tracking mildly-radioactive tracers after the subjects wake up. Further introspection of the data clearly shows that the maximum data-point in the reported range is a statistical outlier. Including the maximum-reported volume in the analysis, the mean aspirated volume comes out to be $0.0345 \mathrm{ml}$ and the median is $0.0215 \mathrm{ml}$. Excluding the outlier, the mean volume revises to $0.021 \mathrm{ml}$ and the median volume adjusts to $0.020 \mathrm{ml}$. The study was based on a total of 10 normal subjects.

Further, while evaluating swallowing mechanisms, it has been reported ${ }^{6}$ that for bolus volumes $5 \mathrm{ml}$ or less, aspirations happen during $13 \%$ of swallows; and for bolus volumes $10 \mathrm{ml}$ or less, aspirations happen during $11 \%$ of swallows. Boluses smaller than $10 \mathrm{ml}$ are associated with silent aspiration ${ }^{9}$ and presumably are the major sources of pathogen-carriers to the deep lungs, and with averaging the reported data, $12 \%$ of the swallowing actions (for $10 \mathrm{ml}$ or smaller bolus volumes) should result in aspiration.

Finally, earlier findings ${ }^{10}$ suggest that a typical person will swallow 500 - 700 times during a day and 24 times during sleep (assuming a standard eight-hour sleep cycle). These numbers thus indicate that a subject will aspirate approximately $12 \%$ of $500-700$ times, i.e. 60 - 84 times during the day, and $12 \%$ of 24 times, i.e. approximately 3 times during sleep.

\subsection{Development of anatomically realistic computational fluid mechanics models}

Allometric relations ${ }^{11}$ show that the minute inhalation is approximately $14.5-20.0 \mathrm{~L} / \mathrm{min}$ for a $65-\mathrm{kg}$ male and $8.8-22.4 \mathrm{~L} / \mathrm{min}$ for a $65-\mathrm{kg}$ female, both for gentle steady breathing. For simplicity, in this study, we have simulated inhalation at only $15 \mathrm{~L} / \mathrm{min}$; the process can be modeled using viscous-laminar steady state flow physics schemes $^{12-21}$.

\subsubsection{Anatomic airway reconstructions}

The in silico anatomic geometries were reconstructed from medical-grade computed tomography (CT) scans sourced from existing de-identified imaging data from two CT-normal subjects. Use of the archived records was approved with exempt status by the Institutional Review Board of the University of North Carolina at Chapel Hill, with the informed consent requirement waived for retrospective computational use. The test subjects include a 61 year-old female (subject for anatomic reconstruction 1, or AR1) and a 37 year-old female (subject for anatomic reconstruction 2, or AR2). As for imaging resolution, the CT slices were collected at coronal depth increments of $0.4 \mathrm{~mm}$. The anatomic airspace was extracted from the scans over a delineation range of -1024 to -300 Hounsfield units, the 
medRxiv preprint doi: https://doi.org/10.1101/2020.12.19.20248544; this version posted December 22, 2020. The copyright holder for this preprint (which was not certified by peer review) is the author/funder, who has granted medRxiv a license to display the preprint in perpetuity. All rights reserved. No reuse allowed without permission.

process was complemented by careful hand-editing of the selected pixels for anatomic accuracy. Subsequently, the reconstructed geometries were spatially meshed into minute volume elements. As per established mesh refinement protocols $^{22,23}$, each computational grid in this study has more than 4 million unstructured, graded tetrahedral elements (namely, 4.54 million in AR1, 4.89 million in AR2).

\subsubsection{Simulating inhalation}

The viscous-laminar flow simulations of the inhalation process were carried out using a segregated solver on ANSYS Fluent, with SIMPLEC pressure-velocity coupling and second-order upwind spatial discretization. We monitored the solution convergence by minimizing the mass continuity and velocity component residuals, and by stabilizing the mass flow rate and static pressure at the airflow outlets. For the pressure-driven flow solutions, typical run-time for 5000 iterations was $5-6$ hours through 4-processor based parallel computations executed at $4.0 \mathrm{GHz}$ speed. We assumed the air density to be $1.204 \mathrm{~kg} / \mathrm{m}^{3}$ and the dynamic viscosity of air to be $1.825 \times 10^{-5} \mathrm{~Pa}$.s.

We enforced the following boundary conditions in the flow simulations: zero velocity (no slip) at the internal airway walls i.e. at the tissues and cartilages enclosing the airway; zero pressure at nostril openings, which acted as the pressure-inlet zones; and negative pressure at the airflow outlet at the base of the nasopharynx, which acted as the pressure-outlet zone. See Fig. 1 for the relative locations of the anatomic regions.

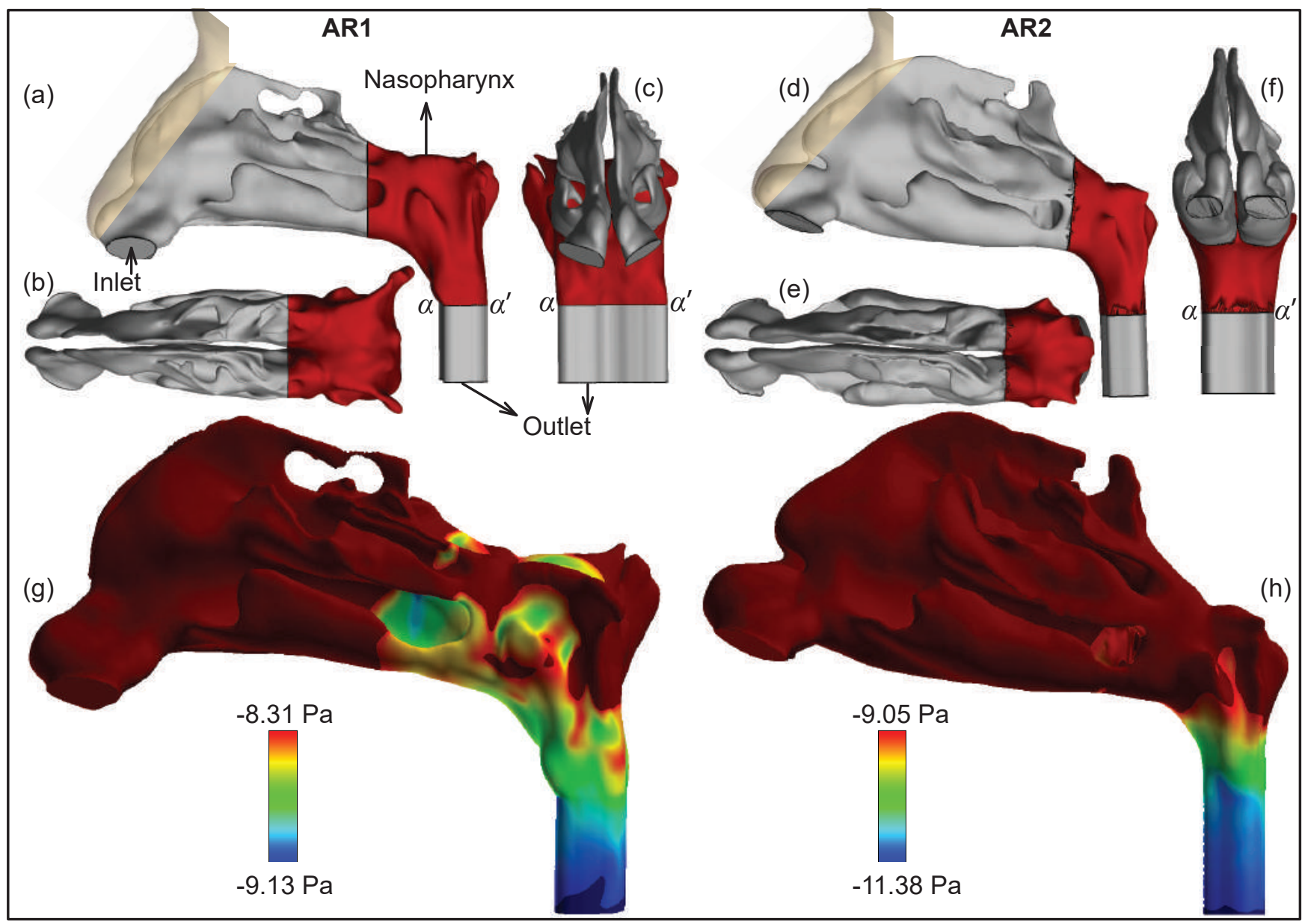

Figure 1. Panels (a), (b), and (c) respectively show the sagittal, axial, and coronal views of the CT-based upper airway domains for anatomic reconstruction 1 (AR1); Panels (d), (e), (f) show the corresponding views for anatomic reconstruction 2 (AR2). Nasopharynx is marked in red. Additionally, $\alpha-\alpha^{\prime}$ shows the location of the wetted perimeter used while estimating the hydraulic radius (see Section 2.2.3). Panel (g) depicts the wall pressure map in AR1, for simulated inhalation of $15 \mathrm{~L} / \mathrm{min}$. Panel (g) shows the wall pressure map for AR1, for simulated inhalation of $15 \mathrm{~L} / \mathrm{min}$. The pressure color-maps for (g) and (h) set the limiting contour colors at the $p_{n}$ (averaged wall pressure at nasopharynx) and $p_{o}$ (averaged pressure at outlet) values that are extracted from the simulated data, in each case. The data post-processing is done on FieldView, under software license provided through the University Partners Program. 
medRxiv preprint doi: https://doi.org/10.1101/2020.12.19.20248544; this version posted December 22, 2020. The copyright holder for this preprint (which was not certified by peer review) is the author/funder, who has granted medRxiv a license to display the preprint in perpetuity.

All rights reserved. No reuse allowed without permission.

\subsubsection{Theoretical estimation of aspirated nasopharyngeal liquid volume}

For an instantaneous pathogenic event such as pharyngeal aspiration, the transport of the nasopharyngeal liquids downstream to the lower airway can be mathematically described through a reduced-order model of steady unidirectional flow. With the assumption of axial symmetry in the airway conduit and no slip boundary condition at the walls, integrating the Navier-Stokes equation for momentum conservation results in

$$
\mathbb{Q}=-\frac{\pi \mathcal{R}^{4}}{8 \mu} \frac{d p}{d z}
$$

where $\mathbb{Q}$ is the instantaneous aspirated volume, $\mathcal{R}$ is the hydraulic radius (cross-section of conduit divided by the perimeter at the nasopharyngeal base), $\mu$ is the sputum viscosity (quantified at 4.59 Poise for mucopurulent ${ }^{24}$ ), and $d p / d z$ is the spatial rate of pressure gradient in the streamwise direction. To adapt the formulation to the present problem, we have post-processed the simulations to extract the averaged wall pressure at the nasopharynx $\left(p_{n}\right)$, since the bolus would typically arise from the shearing action along nasopharyngeal surfaces. We have also extracted the averaged pressure ( $p_{o}$ ) at the outlet (see Fig. 1), located $20 \mathrm{~mm}$ (streamwise $\Delta Z$, so as to ensure full flow development in the simulations) below the nasopharyngeal base. With $\Delta P=p_{o}-p_{n}$, the gradient rate $d p / d z$ can therefore be approximated to simply $\Delta P / \Delta Z$.

\subsection{Data on viral loading}

SARS-CoV-2 is a single-stranded RNA virus, and virological assessments ${ }^{7}$ performed on the sputum of hospitalized COVID-19 patients indicate a mean viral load of $\mathcal{V}_{\text {ave }}=7 \times 10^{6} \mathrm{RNA}$ copies $/ \mathrm{ml}$ of oral fluid. The peak load was $\mathcal{V}_{\text {peak }}=2.35 \times 10^{9}$ copies $/ \mathrm{ml}$.

\section{Results}

3.1 Computational prediction of shear-generated, pressure-driven nasopharyngeal bolus volume With inhalation simulated at $15 \mathrm{~L} / \mathrm{min}$ : for AR1, $\Delta P$ was $-0.82 \mathrm{~Pa}$; for AR2, $\Delta P$ was $2.33 \mathrm{~Pa}$ (see Fig. 1 , Panels $(\mathrm{g})$ and $(\mathrm{h})$ ). The hydraulic radius in AR1 was $3.43 \mathrm{~mm}$, i.e. $\mathcal{R}=0.00343 \mathrm{~m}$; the hydraulic radius in AR2 was $3.06 \mathrm{~mm}$, i.e. $\mathcal{R}=0.00306 \mathrm{~m}$. Consequently using Equation 1, in AR1: the liquid bolus in each aspiration is $\left(\pi \times \mathcal{R}^{4} \times|\Delta P|\right) /(8 \times 0.459 \times 0.02)=4.86 \times 10^{-9} \mathrm{~m}^{3}=0.00486 \mathrm{ml}$. Similarly in AR2: the liquid bolus volume ingested during each aspiration is $0.00874 \mathrm{ml}$.

\subsection{Estimation of aspiration frequency and validation of the computational predictions}

Using the earlier observational data in the first paragraph of Section 2.1, in conjunction with the computed volumes in Section 3.1, the aspiration frequency during each sleep cycle ranges between $0.021 \mathrm{ml} / 0.00874 \mathrm{ml}$ and $0.021 \mathrm{ml} / 0.00486 \mathrm{ml}$, or approximately $2-4$ times. Calculations based on direct experimental observations provide an estimate of 3 aspirations during a sleep cycle (derived in the third paragraph of Section 2.1, using previous reports $\left.{ }^{6,10}\right)$. The computational framework thus provides strong agreement with the direct experimental observations, providing a measure of validation for the underlying fluid dynamics framework.

\subsection{Estimation of virion flow to the lower airway}

Section 2.1 shows that the total number of aspirations of liquid boluses into the lower airway approximately ranges between 63 - 87 times in a day (including the subdued phase during sleep). Based on the data from the current subjects in the numerical simulations (see Section 3.1), the total volume of aspirated liquid in a day is thus between $0.00486 \times 63 \mathrm{ml}$ and $0.00874 \times 87 \mathrm{ml}$, or between $0.3-0.76 \mathrm{ml}$. With the virological data from Section 2.3 , the number of virions penetrating into the lower airway, while suspended in nasopharyngeal fluid boluses, is therefore between $0.3 \times \mathcal{V}_{\text {ave }}=2.1 \times 10^{6}$ and $0.76 \times \mathcal{V}_{\text {ave }}=5.3 \times 10^{6}$, while considering the mean viral load. For peak viral load $\left(\mathcal{V}_{\text {peak }}\right)$ in the sputum, the corresponding number of penetrating virions ranges over $7.1 \times 10^{8}-17.9 \times 10^{8}$. 


\section{Discussion}

We have demonstrated a novel computational approach to estimate virion flow rates to the lower airway from the initial dominant infection zone in the upper airway, i.e. the nasopharynx, which acts as the seeding site for the progressive infection via aspiration of virus-laden boluses of nasopharyngeal fluids. The computational fluid mechanics prediction of the aspirated liquid volume in each occurrence is validated by comparing the number of projected aspirations (approximately $2-4$ ) during an eight-hour sleep cycle with prior observational findings of 3 aspirations in human subjects.

The numerically projected aspiration volumes have been linked to earlier records on aspiration frequency for the entire day, to obtain a total aspirated nasopharyngeal liquid volume of $0.3-0.76 \mathrm{ml}$ each day. Subsequently using sputum assessment data from hospitalized COVID-19 patients, we see that the number of virions penetrating the lower airway ranges over $2.1 \times 10^{6}-5.3 \times 10^{6}$, for mean sputum viral load. When the viral load peaks, the number of penetrating virions increases to $7.1 \times 10^{8}-1.8 \times 10^{9}$. Deep sequencing studies suggest that the minimum infectious dose for SARS-CoV-2 transmission from one human host to another is on the order of $\mathcal{O}\left(10^{2}\right)$ virions $^{25}$ (also supported by our earlier computational findings ${ }^{8}$ ), so it stands to reason that a dose $10^{4}-10^{7}$ times higher will suffice to seed a second infection site within the same host, particularly given the relatively high levels of ACE2 expression in a subset of alveolar cells ${ }^{26}$.

Our results should be interpreted as being preliminary, given that the numerical findings are based on simulated data from only two CT-normal subjects. We note that there is good agreement between numerical predictions of aspiration frequency with earlier observational findings, which speaks to the validity of the underlying computational and mathematical framework. The size of the projected viral dose also speaks to the robustness of the conclusion - it is likely that virion flow from the nasopharynx to the lungs occurs in large excess of the minimum dose required to seed a lung infection for many individuals.

Our findings suggest a simple aspiration-based physiological mechanism for COVID-19 etiology following initial SARS-CoV-2 infection in the nasopharynx. Such a mechanistic link may be valuable in identifying risk factors that predispose patients to progress to COVID-19 disease following SARS-CoV-2 infection. For example, a prediction that flows readily (pun intended) from this proposed mechanism is that individuals with dysphagia (problems initiating swallowing) may be at increased risk of developing COVID-19 following SARS-CoV-2 infection, and may have more negative outcomes with the disease.

One condition associated with dysphagia is obstructive sleep apnea (OSA $)^{27-29}$, with increased nocturnal aspiration $^{30}$ and risk of aspiration pneumonia ${ }^{31}$. Based on the mechanism proposed by us for viral spread to the lungs, individuals with OSA would thus be expected to be at a higher risk for COVID-19. In fact, this has been reported by a number of different investigators ${ }^{32-36}$. One study, for example, reported an association of OSA with increased risk for hospitalization (OR 1.65; 95CI $(1.36,2.02))$ and respiratory failure (OR 1.98; 95CI $(1.65,2.37)$ ) due to COVID-19, after adjusting for diabetes, hypertension, and body mass index ${ }^{37}$. As a practical matter, our work suggests that individuals with OSA should not suspend the use of their CPAP devices upon testing positive for SARS-CoV-2, as has been suggested by some practitioners ${ }^{38}$.

As the prevalence of dysphagia also increases with other factors such as increased age ${ }^{39}$, cancer treatment ${ }^{40}$, and Parkinsonism ${ }^{41}$, the physiological mechanism proposed here may also account for some of the documented increased risk of adverse outcomes ${ }^{42-45}$ in these groups. Further study is required to understand the importance of dysphagia in general as a predictive factor for adverse outcomes with COVID-19.

Our findings also point to the critical importance of reducing SARS-CoV-2 viral load in the early stages of the infection. Therapeutics and prophylactics that fail to prevent the virus from gaining a foothold in the nasopharynx will allow the escalation of the disease in at least a subset of the population, which may already be more vulnerable to adverse outcomes from the disease. If vaccinal prophylaxis does not provide sterilizing immunity, then vaccinated individuals infected with SARS-CoV-2 in the nasopharynx may be subjected to a steady stream of virions testing their immune system with potential immune-evading mutations. Such a scenario is of particular concern for antibody prophylactics and vaccines that target the spike protein, work by us ${ }^{46}$ and others ${ }^{47,48}$ suggests that the spike protein has a relatively high mutational tolerance, and is expected to readily generate immune-evading mutations when 
antibody-based prophylaxis becomes widespread. In this scenario, some proportion of patients may experience breakthrough infections as a result of evolutionarily-mediated resistance, and seed transmission chains of antibodyor vaccine-resistant SARS-CoV-2 within the population. Biomedical interventions that rely on limiting the spread of the virus, that has established itself within the body, may underestimate the systemic threat that nasopharyngeal SARS-CoV-2 infections pose to individual patients as well as to public health.

\section{Data availability}

We have generated simulated, quantitative, de-identified data on the flow physics in respiratory physiology. The data-sets (including Fluent .cas and .dat files) and the numeric protocols; along with MATLAB codes, Wolfram Mathematica notebooks, and Microsoft Excel spreadsheets used for data post-processing - are available on-request from the lead corresponding author (SB).

\section{Funding}

This material is based upon work partially supported by the National Science Foundation (NSF) RAPID Grant 2028069 for COVID-19 research, with SB as the Principal Investigator. Any opinions, findings, and conclusions or recommendations expressed here are, however, those of the author and do not necessarily reflect NSF's views.

\section{Acknowledgments}

The work is the result of an ongoing collaboration between SB (Assistant Professor at SDSU and Visiting Scholar at UNC Chapel Hill) and AC (Chief Executive Officer at Fractal Therapeutics). Computing facilities at both SDSU and UNC Chapel Hill have been used for this project. The authors also acknowledge Prof. Sunghwan Jung (at Cornell University), Prof. Leonardo Chamorro (at University of Illinois at Urbana-Champaign), and Prof. Diane Joseph-McCarthy (at Boston University) for several fruitful discussions.

\section{References}

1. Hou, Y. J. et al. SARS-CoV-2 Reverse Genetics Reveals a Variable Infection Gradient in the Respiratory Tract. Cell (2020).

2. Matheson, N. J. \& Lehner, P. J. How does SARS-CoV-2 cause COVID-19? Science 369, 510-511 (2020).

3. Mittal, R., Ni, R. \& Seo, J. H. The flow physics of COVID-19. J. Fluid Mech. 894 (2020).

4. Patel, M. R. et al. Performance of oropharyngeal swab testing compared to nasopharyngeal swab testing for diagnosis of COVID-19. Clin. Infect. Dis. (2020).

5. Gleeson, K., Maxwell, S. L. \& Eggli, D. F. Quantitative aspiration during sleep in normal subjects. Chest 111, 1266-1272 (1997).

6. Butler, S. G. et al. Factors influencing aspiration during swallowing in healthy older adults. The Laryngoscope 120, 2147-2152 (2010).

7. Wölfel, R. et al. Virological assessment of hospitalized patients with COVID-2019. Nature 581, 465-469 (2020).

8. Basu, S. Computational characterization of inhaled droplet transport in the upper airway leading to SARS-CoV-2 infection. medRxiv: preprint server for health sciences (2020).

9. Leder, S. B., Suiter, D. M. \& Green, B. G. Silent aspiration risk is volume-dependent. Dysphagia 26, 304-309 (2011). 
10. Speech-Pathology-Australia. Swallowing Awareness Day. Web link (accessed 16-December-2020).

11. Garcia, G. J. M. et al. Dosimetry of nasal uptake of water-soluble and reactive gases: a first study of interhuman variability. Inhalation Toxicol. 21, 607-618 (2009).

12. Inthavong, K. et al. Geometry and airflow dynamics analysis in the nasal cavity during inhalation. Clin. Biomech. 66, 97-106 (2019).

13. Zhang, Y. et al. Computational investigation of dust mite allergens in a realistic human nasal cavity. Inhalation Toxicol. 31, 224-235 (2019).

14. Basu, S., Frank-Ito, D. O. \& Kimbell, J. S. On computational fluid dynamics models for sinonasal drug transport: Relevance of nozzle subtraction and nasal vestibular dilation. Int. J. for Numer. Methods Biomed. Eng. 34, e2946 (2018).

15. Farzal, Z. et al. Comparative study of simulated nebulized and spray particle deposition in chronic rhinosinusitis patients. In International Forum of Allergy \& Rhinology, vol. 9, 746-758 (Wiley Online Library, 2019).

16. Kiaee, M., Wachtel, H., Noga, M. L., Martin, A. R. \& Finlay, W. H. Regional deposition of nasal sprays in adults: A wide ranging computational study. Int. J. for Numer. Methods Biomed. Eng. 34, e2968 (2018).

17. Zhao, K., Scherer, P. W., Hajiloo, S. A. \& Dalton, P. Effect of anatomy on human nasal air flow and odorant transport patterns: implications for olfaction. Chem. Senses 29, 365-379 (2004).

18. Xi, J. \& Longest, P. W. Numerical predictions of submicrometer aerosol deposition in the nasal cavity using a novel drift flux approach. Int. J. Heat Mass Transf. 51, 5562-5577 (2008).

19. Shanley, K. T., Zamankhan, P., Ahmadi, G., Hopke, P. K. \& Cheng, Y. S. Numerical simulations investigating the regional and overall deposition efficiency of the human nasal cavity. Inhalation Toxicol. 20, 1093-1100 (2008).

20. Kelly, J. T., Prasad, A. K. \& Wexler, A. S. Detailed flow patterns in the nasal cavity. J. Appl. Physiol. 89, 323-337 (2000).

21. Basu, S. et al. Numerical evaluation of spray position for improved nasal drug delivery. Sci. Reports 10, 1-18 (2020).

22. Frank-Ito, D. O., Wofford, M., Schroeter, J. D. \& Kimbell, J. S. Influence of mesh density on airflow and particle deposition in sinonasal airway modeling. J. Aerosol Medicine Pulm. Drug Deliv. 29, 46-56 (2016).

23. Basu, S., Witten, N. \& Kimbell, J. S. Influence of localized mesh refinement on numerical simulations of post-surgical sinonasal airflow. J. Aerosol Medicine Pulm. Drug Deliv. 30, A-14 (2017).

24. Lopez-Vidriero, M. T., Charman, J., Keal, E., De Silva, D. J. \& Reid, L. Sputum viscosity: correlation with chemical and clinical features in chronic bronchitis. Thorax 28, 401-408 (1973).

25. Wang, D. et al. Population Bottlenecks and Intra-host Evolution during Human-to-Human Transmission of SARS-CoV-2. bioRxiv (2020).

26. Ortiz, M. E. et al. Heterogeneous expression of the SARS-Coronavirus-2 receptor ACE2 in the human respiratory tract. EBioMedicine 60, 102976 (2020).

27. Sato, K., Chitose, S. I., Sato, F. \& Umeno, H. Deglutition and respiratory patterns during sleep in the aged with OSAS. Laryngoscope Investig. Otolaryngol. 3, 500-506 (2018).

28. Bhutada, A. M., Broughton, W. A. \& Focht Garand, K. L. Obstructive sleep apnea syndrome (OSAS) and swallowing function - a systematic review. Sleep Breath. 1-9 (2020).

29. Valbuza, J. S. et al. Swallowing dysfunction related to obstructive sleep apnea: a nasal fibroscopy pilot study. Sleep Breath. 15, 209-213 (2011).

30. Sato, K. et al. Sleep-related deglutition and respiratory phase patterns in the aged with obstructive sleep apnea under CPAP therapy. Acta Oto-Laryngologica 1-8 (2020). 
medRxiv preprint doi: https://doi.org/10.1101/2020.12.19.20248544; this version posted December 22, 2020. The copyright holder for this preprint (which was not certified by peer review) is the author/funder, who has granted medRxiv a license to display the preprint in perpetuity. All rights reserved. No reuse allowed without permission.

31. Sato, K. et al. Recurrent aspiration pneumonia precipitated by obstructive sleep apnea. Auris Nasus Larynx (2020).

32. McSharry, D. \& Malhotra, A. Potential influences of obstructive sleep apnea and obesity on COVID-19 severity. J. Clin. Sleep Medicine 16, 1645-1645 (2020).

33. Bhatraju, P. K. et al. COVID-19 in critically ill patients in the Seattle region - case series. New Engl. J. Medicine 382, 2012-2022 (2020).

34. Arentz, M. et al. Characteristics and outcomes of 21 critically ill patients with COVID-19 in Washington State. JAMA 323, 1612-1614 (2020).

35. Tufik, S., Gozal, D., Ishikura, I. A., Pires, G. N. \& Andersen, M. L. Does obstructive sleep apnea lead to increased risk of COVID-19 infection and severity? J. Clin. Sleep Medicine jcsm-8596 (2020).

36. Cade, B. E., Dashti, H. S., Hassan, S. M., Redline, S. \& Karlson, E. W. Sleep Apnea and COVID-19 mortality and hospitalization. Am. J. Respir. Critical Care Medicine 202, 1462-1464 (2020).

37. Maas, M. B., Kim, M., Malkani, R. G., Abbott, S. M. \& Zee, P. C. Obstructive Sleep Apnea and Risk of COVID-19 Infection, Hospitalization and Respiratory Failure. Sleep Breath. 1-3 (2020).

38. Cleveland-Clinic. Can PAP Therapy Machines Increase the Risk of Spreading the Coronavirus? Web link (accessed 18-December-2020).

39. Aslam, M. \& Vaezi, M. F. Dysphagia in the elderly. Gastroenterol. \& Hepatol. 9, 784 (2013).

40. Raber-Durlacher, J. E. et al. Swallowing dysfunction in cancer patients. Support. Care Cancer 20, 433-443 (2012).

41. Suttrup, I. \& Warnecke, T. Dysphagia in Parkinson's disease. Dysphagia 31, 24-32 (2016).

42. Rutten, J. J. S. et al. Clinical Suspicion of COVID-19 in Nursing Home residents: symptoms and mortality risk factors. J. Am. Med. Dir. Assoc. 21, 1791-1797 (2020).

43. Vignatelli, L. et al. Risk of hospitalization and death for COVID-19 in people with Parkinson's disease or Parkinsonism. Mov. Disord. (2020).

44. for-Disease-Control-and Prevention, C. COVID-19 Hospitalization and Death by Age. Web link (accessed 18-December-2020).

45. Kuderer, N. M. et al. Clinical impact of COVID-19 on patients with cancer (CCC19): a cohort study. The Lancet (2020).

46. Van Egeren, D. et al. Risk of evolutionary escape from neutralizing antibodies targeting SARS-CoV-2 spike protein. medRxiv: preprint server for health sciences (2020).

47. Thomson, E. C. et al. The circulating SARS-CoV-2 spike variant N439K maintains fitness while evading antibody-mediated immunity. bioRxiv (2020).

48. Starr, T. N. et al. Deep mutational scanning of SARS-CoV-2 receptor binding domain reveals constraints on folding and ACE2 binding. Cell 182, 1295-1310 (2020). 